\title{
Analyticity and unitarity constraints on form factors
}

\section{Irinel Caprini ${ }^{* \dagger}$}

Horia Hulubei National Institute for Physics and Nuclear Engineering, P.O.B. MG-6, 077125

Bucharest-Magurele, Romania

E-mail: caprini@theory.nipne.ro

\begin{abstract}
After a brief historical review of the method of unitarity bounds for the hadronic form factors, proposed by Okubo more than 40 years ago, I present the mathematical foundation of the formalism known as the Meiman problem and its generalizations. It allows the optimal implementation of the available information on the phase and modulus of the form factors on the unitarity cut, and leads to upper and lower bounds on the values of the form factors and their derivatives at points inside the holomorphy domain. The formalism is useful for checking the consistency of experimental data and theoretical predictions, in particular from Chiral Perturbation Theory and lattice calculations. It provides also means for controlling the truncation error in parametrizations of the form factors of interest for precision predictions in flavour physics. Several recent applications to the pion electromagnetic form factor and the form factors relevant for the semileptonic decays $D \rightarrow \pi l v$ and $B \rightarrow \pi l v$ are discussed.
\end{abstract}

The 7th International Workshop on Chiral Dynamics,

August 6 -10, 2012

Jefferson Lab, Newport News, Virginia, USA

\footnotetext{
* Speaker.

${ }^{\dagger}$ Talk based on works done in collaboration with G. Abbas, B. Ananthanarayan, C. Bourrely, D. Das, I. Sentitemsu Imsong, L. Lellouch and S. Ramanan.
} 


\section{Introduction}

In this talk I will consider electromagnetic form factors $F_{P}(t)$ of light pseudoscalar mesons $P$ (in particular $P=\pi$ ), defined as

$$
\left\langle P^{+}\left(p^{\prime}\right)\left|J_{\mu}^{\mathrm{elm}}\right| P^{+}(p)\right\rangle=\left(p+p^{\prime}\right)_{\mu} F_{P}(t),
$$

and form factors relevant for the weak semileptonic transitions $P_{1} \rightarrow P_{2} \ell v$ (where $P_{1}=B, D$ or $K$ and $P_{2}=D$ or $\pi$ ), appearing in the matrix element

$$
\left\langle P_{2}^{+}\left(p^{\prime}\right)\left|J_{\mu}^{\text {weak }}\right| P_{1}^{0}(p)\right\rangle=\left(p^{\prime}+p\right)_{\mu} f_{+}(t)+\left(p-p^{\prime}\right)_{\mu} f_{-}(t) .
$$

Here $f_{+}(t)$ is the vector form factor and $f_{0}(t)=f_{+}(t)+\frac{t}{M_{P_{1}}^{2}-M_{P_{2}}^{2}} f_{-}(t)$ is the so-called scalar form factor. In the general discussion I shall denote a generic hadronic form factor by $F(t)$.

At low energies the theoretical description of the hadronic form factors is based on Chiral Perturbation Theory (ChPT), lattice QCD and various types of QCD Sum Rules, while perturbative QCD is valid at high momentum transfers of spacelike type, $t=-Q^{2}<0$, where it predicts in general an asymptotic $1 / Q^{2}$ scaling. At intermediate energies a consistent description does not exist, the theoretical models proposed in the literature being affected by large uncertainties.

Analyticity and unitarity have been much exploited in phenomenological studies of form factors, especially in the early sixties, when enthusiasts of analytic S-matrix theory were quoted ${ }^{1}$ as saying that "one of the most remarkable discoveries in elementary particle physics has been that of the existence of the complex plane". The question is whether these properties are still useful at present, when a successful theory of strong interactions exists. The goal of this talk is to show that, suitably exploited, analyticity and unitarity provide useful tools as a link between various descriptions and for making precision predictions at low energies.

The analytic properties of form factors are in general much simpler than those of the scattering amplitudes (complications like unphysical regions or anomalous thresholds are not encountered in the cases considered). Causality implies that a generic form factor $F(t)$ is a real analytic function, $F\left(t^{*}\right)=F^{*}(t)$, in the complex $t$-plane with a cut along the real axis from the specific lowest unitarity threshold $t_{+}$to infinity. The discontinuity across the cut is given by unitarity, which in the elastic region reads

$$
\operatorname{Im} F(t+i \varepsilon)=\theta\left(t-t_{+}\right) \sigma(t)\left(f_{J}^{I}(t)\right)^{*} F(t), \quad t<t_{\text {in }},
$$

where $\sigma(t)=\sqrt{1-t_{+} / t}$ is the two particle phase space, $f_{J}^{I}(t)=\left(e^{2 i \delta_{J}^{I}(t)}-1\right) /(2 i \sigma(t))$ is the partial wave amplitude with the same spin $J$ and isospin $I$ quantum numbers, and $t_{\text {in }}$ is the first inelastic threshold. This implies in particular Fermi-Watson theorem:

$$
\arg [F(t+i \varepsilon)]=\delta_{J}^{I}(t), \quad t_{+} \leq t \leq t_{\text {in }},
$$

where $\delta_{I}^{J}(t)$ is the phase-shift of the corresponding partial wave of elastic scattering.

The form factors are analytic at $t=0$, where they admit Taylor expansions convergent inside the circle passing through the nearest singularities. These expansions are written usually as

$$
F(t)=1+\frac{1}{6}\left\langle r_{\pi}^{2}\right\rangle t+c t^{2}+d t^{3}+\cdots
$$

\footnotetext{
${ }^{1}$ J. Schwinger in Particles, Sources and Fields, vol. 1, page 36, Addison Wesley, 1970.
} 
for the pion electromagnetic form factor, where $\left\langle r_{\pi}^{2}\right\rangle$ denotes the charge radius squared, and

$$
f_{k}(t)=f_{k}(0)\left(1+\lambda_{k}^{\prime} \frac{t}{M^{2}}+\frac{1}{2} \lambda_{k}^{\prime \prime} \frac{t^{2}}{M^{4}}+\cdots\right), \quad k=+, 0,
$$

for the weak form factors, where $M$ is a suitable mass scale.

There are three known types of integral representations which exploit analyticity and allow the analytic continuation of the form factors from the cut to points inside the analyticity domain: the standard dispersion relation, based on Cauchy integral and the reality property, written (modulo subtractions) as

$$
F(t)=\frac{1}{\pi} \int_{t_{+}}^{\infty} \frac{\operatorname{Im} F\left(t^{\prime}+i \varepsilon\right) d t^{\prime}}{t^{\prime}-t}
$$

the Omnès representation, which expresses the function in terms of its phase

$$
F(t)=P(t) \exp \left(\frac{t}{\pi} \int_{t_{+}}^{\infty} d t^{\prime} \frac{\delta\left(t^{\prime}\right)}{t^{\prime}\left(t^{\prime}-t\right)}\right), \quad \delta(t) \equiv \arg F(t+i \varepsilon),
$$

where $P(t)$ is an arbitrary polynomial accounting for the zeros of $F(t)$ at points $t_{i}$ in the complex plane, $P\left(t_{i}\right)=0$, and the representation in terms of the modulus

$$
F(t)=B(t) \exp \left(\frac{\sqrt{t_{+}-t}}{\pi} \int_{t_{+}}^{\infty} \frac{\ln \left|F\left(t^{\prime}\right)\right| \mathrm{d} t^{\prime}}{\sqrt{t^{\prime}-t_{+}}\left(t^{\prime}-t\right)}\right)
$$

where $B(t)$ is a so-called Blaschke factor, with the property $|B(t)|=1$ for $t>t_{+}$, which also accounts for the possible zeros at points $t_{i}, B\left(t_{i}\right)=0$.

None of these standard representations has complete input: the imaginary part is not directly measurable, the phase $\delta(t)$ is sometimes known from Fermi-Watson theorem in the elastic region, but is unknown for $t>t_{\text {in }}$, while the modulus $|F(t)|$ is measured directly in some cases, but only on a limited energy range. Moreover, both the phase and modulus representations require the positions of the zeros in the complex plane, which are not known. Various analytic parametrizations are also often employed, but they have in general little predictive power outside their original range, due to the phenomenon known as "instability of analytic continuation" [1].

In this context, it is remarkable that Okubo [2], back in the early 70', devised an approach which produced almost model-independent constraints on the $K_{\ell 3}$ form factors in the physical region of $K \rightarrow \pi \ell v$ decays. The method starts with a polarization tensor of a suitable operator, calculated from current algebra at spacelike momenta, and exploits unitarity and positivity of the spectral functions in the dispersion relations valid for the invariant amplitudes. These steps lead to an upper bound on a weighted integral along the unitarity cut of the modulus squared of a related form factor. From this condition, mathematical techniques of complex analysis [3, 4] allow one to derive bounds on the values of the form factor and its derivatives at points inside the analyticity domain. Okubo approach, known also as "method of unitarity bounds", was further applied by Micu [5], Auberson et al. [6], Singh and Raina [7] and other authors.

A modern version of the approach was put forward in 1981 by Bourrely, Machet and de Rafael [8], who obtained the input spacelike correlators from perturbative QCD and Operator Product Expansion (OPE) rather than from current algebra. Later on, de Rafael and Taron [9] made a first application to the semileptonic decays of heavy quarks in the context of Heavy Quark Effective 
Theory (HQET) and derived bounds on the slope of the universal Isgur-Wise function. These papers opened the way to many applications to the pion electromagnetic form factor and form factors describing the semileptonic decays $B \rightarrow D^{(*)} \ell v, B \rightarrow \pi \ell v, D \rightarrow \pi \ell v$ and $K \rightarrow \pi \ell v$ (see [10]-[24] and references therein). Also, suitable mathematical techniques were developed for covering more complex situations, determined by the theoretical and experimental progress (for recent reviews see $[25,26])$.

In the present talk I give a brief presentation of these techniques and their applications. After a short introduction to the standard Okubo approach, I discuss in Section 3 three functional extremal problems related to this approach and its generalizations. In Section 4, I present several applications to the pion electromagnetic form factor and the form factors relevant for the semileptonic decays $D \rightarrow \pi l v$ and $B \rightarrow \pi l v$, leaving the presentation of $K \rightarrow \pi l v$ to another talk at this Workshop [27]. A few concluding remarks are given in the final section.

\section{Okubo approach}

I illustrate the procedure in its modern version applied for the first time in [8]. We start with the polarization tensor of the weak current $J_{\mu}^{\text {weak }}$ relevant for the transition $P_{1} \rightarrow P_{2} \ell v$ :

$$
i \int d^{4} x e^{i q \cdot x}\left\langle 0\left|T\left\{J_{\mu}^{\text {weak }}(x) J_{v}^{\text {weak }}(0)^{\dagger}\right\}\right| 0\right\rangle=\left(-g_{\mu v} q^{2}+q_{\mu} q_{v}\right) \Pi_{1}\left(q^{2}\right)+q_{\mu} q_{v} \Pi_{0}\left(q^{2}\right),
$$

and write unsubtracted dispersion relations for the correlators:

$$
\begin{gathered}
\chi_{1}\left(Q^{2}\right) \equiv-\frac{1}{2} \frac{\partial^{2}}{\partial\left(Q^{2}\right)^{2}}\left[Q^{2} \Pi_{1}\left(-Q^{2}\right)\right]=\frac{1}{\pi} \int_{0}^{\infty} d t \frac{t \operatorname{Im} \Pi_{1}(t)}{\left(t+Q^{2}\right)^{3}} \\
\chi_{0}\left(Q^{2}\right) \equiv \frac{\partial}{\partial Q^{2}}\left[Q^{2} \Pi_{0}\left(-Q^{2}\right)\right]=\frac{1}{\pi} \int_{0}^{\infty} d t \frac{t \operatorname{Im} \Pi_{0}(t)}{\left(t+Q^{2}\right)^{2}} .
\end{gathered}
$$

Unitarity and positivity connect the spectral functions of the above dispersion relations to the weak form factors defined in (1.2). Namely, keeping two-body states in the unitarity sum leads to the inequalities

$$
\begin{aligned}
\operatorname{Im} \Pi_{1}(t) & \geq \frac{3}{2} \frac{1}{48 \pi} \frac{\left[\left(t-t_{+}\right)\left(t-t_{-}\right)\right]^{3 / 2}}{t^{3}}\left|f_{+}(t)\right|^{2}, \\
\operatorname{Im} \Pi_{0}(t) & \geq \frac{3}{2} \frac{t_{+} t_{-}}{16 \pi} \frac{\left[\left(t-t_{+}\right)\left(t-t_{-}\right)\right]^{1 / 2}}{t^{3}}\left|f_{0}(t)\right|^{2},
\end{aligned}
$$

where $t_{ \pm}=\left(M_{P_{1}} \pm M_{P_{2}}\right)^{2}$. By inserting these inequalities into the dispersion relations (2.2) and (2.3), one obtains the generic relation

$$
\frac{1}{\pi} \int_{t_{+}}^{\infty} \rho(t)|F(t)|^{2} \mathrm{~d} t \leq I,
$$

where $\rho(t)$ is a definite weight and $I$ is calculated from perturbative QCD and OPE for the corresponding correlators (we omitted for simplicity the dependence on $Q^{2}$ of $\rho$ and $I$ ). To ensure the validity of OPE, the spacelike momentum $Q^{2}$ must be taken sufficiently large for light mesons $P_{1}$ and $P_{2}$, while the choice $Q^{2}=0$ is reasonable for heavy-heavy or heavy-light form factors.

A comment about the connection of the relation (2.6) with the stability of analytic continuation is of interest. As is known, analyticity has two facets, which may be referred to as its "splendour" 
and its "dangers". They are related to the fact that analytic continuation is unique, but it is also an unstable (ill-posed) problem in the Hadamard sense. This means that two analytic functions very close along a range $\Gamma$ in the complex plane may differ arbitrarily much outside $\Gamma$. There are many phenomenological consequences of this instability: a notorious example is the poor determination of resonances deep in the complex energy plane (like $\sigma$ or $f_{0}(500)$ in $\pi \pi$ scattering) from BreitWigner parametrizations valid on the real axis.

A known mathematical result (known as Tikhonov regularization) stipulates that the analytic continuation is stabilized if the class of admissible functions forms a compact set. It can be shown (see Ciulli et al. [1]) that the $L^{2}$-norm condition (2.6) derived from Okubo approach defines a compact set in the Hardy space $H^{2}$ of analytic functions with finite $L^{2}$-norm on the boundary [4], and plays the role of a stabilizing condition which ensures the stability (i.e. the continuity with respect to the input variation) of the extrapolation to points inside the holomorphy domain.

For the present discussion the crucial fact is that from condition (2.6) one can derive explicit constraints on the values of the form factors at interior points and on the parameters of Taylor expansions like (1.5) and (1.6). Mathematically, the problem belongs to the analytic interpolation theory for functions in the Hardy class $H^{2}[3,4]$ and is known to physicists as the "Meiman problem" [3]. In the next section we shall discuss three versions of physical interest.

\section{Meiman problem and its generalizations}

Problem 1: From the $L^{2}$-norm condition (2.6) find constraints on the values $F\left(t_{n}\right)$ and the derivatives $F^{(k)}\left(t_{j}\right)$ at some real or complex points on the first Riemann sheet of the complex $t$ plane outside the unitarity cut.

The problem is written in a canonical form by performing the conformal mapping

$$
\tilde{z}\left(t, t_{0}\right)=\frac{\sqrt{t_{+}-t_{0}}-\sqrt{t_{+}-t}}{\sqrt{t_{+}-t_{0}}+\sqrt{t_{+}-t}}
$$

which maps the $t$-plane cut for $t>t_{+}$onto the unit disk $|z|<1$, where $z \equiv \tilde{z}\left(t, t_{0}\right)$. Here $t_{0}<t_{+}$is an arbitrary parameter, denoting the point mapped onto the origin, $\tilde{z}\left(t_{0}, t_{0}\right)=0$.

Consider further a so-called outer function [4], i.e. a function analytic and without zeros in $|z|<1$, with modulus squared on $|z|=1$ equal to $\rho(t)|d \tilde{t} / d z|$, where $t \equiv \tilde{t}\left(z, t_{0}\right)$ is the inverse of (3.1). Denoting by $w(z)$ this function, it is written in general in terms of its modulus on the boundary as ${ }^{2}$

$$
w(z)=\exp \left[\frac{1}{2 \pi} \int_{0}^{2 \pi} \mathrm{d} \theta \frac{e^{i \theta}+z}{e^{i \theta}-z} \ln \left[\rho\left(\tilde{t}\left(e^{i \theta}, t_{0}\right)\right)|d \tilde{t} / d z|\right]\right] .
$$

Then the function $g(z)$ defined by

$$
g(z)=F\left(\tilde{t}\left(z, t_{0}\right)\right) w(z)
$$

is analytic in $|z|<1$ and satisfies the inequality

$$
\frac{1}{2 \pi} \int_{0}^{2 \pi}\left|g\left(e^{i \theta}\right)\right|^{2} d \theta \leq I
$$

\footnotetext{
${ }^{2}$ In many cases of physical interest the function $w(z)$ has a simple analytic expression, see for instance $[8,13,25,22]$.
} 
With techniques of complex analysis [3, 4], one can show that (3.4) implies the positivity of the following determinant and of its minors:

$$
\left|\begin{array}{ccccc}
\bar{I} & \bar{\xi}_{1} & \bar{\xi}_{2} & \cdots & \bar{\xi}_{N} \\
\bar{\xi}_{1} & \frac{z_{1}^{2 K}}{1-z_{1}^{2}} & \frac{\left(z_{1} z_{2}\right)^{K}}{1-z_{1} z_{2}} & \cdots & \frac{\left(z_{1} z_{N}\right)^{K}}{1-z_{1} z_{N}} \\
\vdots & \vdots & \vdots & \vdots & \vdots \\
\bar{\xi}_{N} & \frac{\left(z_{1} z_{N}\right)^{K}}{1-z_{1} z_{N}} & \frac{\left(z_{2} z_{N}\right)^{K}}{1-z_{2} z_{N}} & \cdots & \frac{z_{N}^{2 K}}{1-z_{N}^{2}}
\end{array}\right| \geq 0
$$

where $\bar{I}=I-\sum_{k=0}^{K-1} g_{k}^{2}$ and $\bar{\xi}_{n}=g\left(z_{n}\right)-\sum_{k=0}^{K-1} g_{k} z_{n}^{k}$ are defined in terms of the values (for simplicity we restricted to real points $z_{n}$ and the derivatives at $z=0$ ):

$$
\left[\frac{1}{k !} \frac{d^{k} g(z)}{d z^{k}}\right]_{z=0}=g_{k}, \quad 0 \leq k \leq K-1, \quad g\left(z_{n}\right)=\xi_{n}, \quad z_{n}=z_{n}^{*}, \quad 1 \leq n \leq N .
$$

By using (3.3) one can express the inequality (3.5) as a quadratic constraint on the values of the form factor $F(t)$ and its derivatives. From this, upper and lower bounds on $F\left(t_{n}\right)$ at an arbitrary point $t_{n}$ included in the set (3.6) are easily obtained by solving quadratic equations.

It is possible to implemented exactly also the phase in the elastic region, if it is available through the Fermi-Watson theorem (1.4) and the known phase-shift of the related scattering amplitude. The corresponding extremal problem is:

Problem 2: From the relations (1.4) and (2.6), find constraints on the values $F\left(t_{n}\right)$ and the derivatives $F^{(k)}\left(t_{j}\right)$ at some real or complex points on the first Riemann sheet outside the cut.

The problem can be solved exactly with standard techniques of functional optimization, which lead to an integral equation of Fredholm type for a generalized Lagrange multiplier. The general solution is given in $[25,26]$.

In physical situations, the condition (2.6) is sometimes replaced by the inequality

$$
\frac{1}{\pi} \int_{t_{\text {in }}}^{\infty} \rho(t)|F(t)|^{2} d t \leq I^{\prime},
$$

where $t_{\text {in }}$ is the first inelastic threshold and $I^{\prime}$ is a known quantity. We can formulate then the following extremal problem:

Problem 3: From the relations (1.4) and (3.7), find constraints on the values $F\left(t_{n}\right)$ and the derivatives $F^{(k)}\left(t_{j}\right)$ at some real or complex points on the first Riemann sheet outside the cut.

Note that the integral (3.7) from $t_{\text {in }}$ to infinity is not available directly from Okubo approach. If some data on the modulus below $t_{\text {in }}$ are available, the quantity $I^{\prime}$ can be estimated by subtracting the integral on the range $\left(t_{+}, t_{\text {in }}\right)$ from the total integral (2.6), obtained from Okubo approach. For the pion electromagnetic form factor, the recent high-statistics data on the modulus on the cut allow a direct evaluation of the integral (3.7) for suitable choices of the weight $\rho(t)$, thus superseding the Okubo approach.

The solution to Problem 3 is found by defining first the Omnès function (for $t>t_{\text {in }}$, the unknown phase $\delta(t) \equiv \arg [F(t+i \varepsilon)]$ is taken as an arbitrary smooth function):

$$
\mathscr{O}(t)=\exp \left(\frac{t}{\pi} \int_{t_{+}}^{\infty} d t \frac{\delta\left(t^{\prime}\right)}{t^{\prime}\left(t^{\prime}-t\right)}\right)
$$


and define the function $h(t)$ by $F(t)=\mathscr{O}(t) h(t)$, which is obviously real below $t_{\text {in }}$, i.e. is analytic in the $t$-plane cut only for $t>t_{\text {in. }}$. Then the condition (3.7) is written as

$$
\frac{1}{\pi} \int_{t_{\text {in }}}^{\infty} \rho(t)|\mathscr{O}(t)|^{2}|h(t)|^{2} d t \leq I^{\prime}
$$

Thus $h(t)$ satisfies the conditions of Problem 1, with two modifications: the $t$-plane cut for $t>t_{+}$ is replaced by the $t$-plane cut for $t>t_{\text {in }}$, and the weight $\rho(t)$ is replaced by $\rho(t)|\mathscr{O}(t)|^{2}$. Therefore the solution can be written in the form (3.5), where now the conformal mapping reads

$$
\tilde{z}\left(t, t_{0}\right)=\frac{\sqrt{t_{\text {in }}-t_{0}}-\sqrt{t_{\text {in }}-t}}{\sqrt{t_{\text {in }}-t_{0}}+\sqrt{t_{\text {in }}-t}}, \quad \tilde{z}\left(t_{0}, t_{0}\right)=0
$$

and the function $g(z)$ is defined by:

$$
g(z) \equiv F\left(\tilde{t}\left(z, t_{0}\right)\right)\left[\mathscr{O}\left(\tilde{t}\left(z, t_{0}\right)\right)\right]^{-1} w(z) \omega(z),
$$

involving the Omnès function $\mathscr{O}(t)$ defined in (3.8) and the two outer functions [13, 25]

$$
w(z)=\exp \left[\frac{1}{2 \pi} \int_{0}^{2 \pi} \mathrm{d} \theta \frac{e^{i \theta}+z}{e^{i \theta}-z} \ln \left[\rho\left(\tilde{t}\left(e^{i \theta}, t_{0}\right)\right)|d \tilde{t} / d z|\right]\right]
$$

and

$$
\omega(z)=\exp \left(\frac{\sqrt{t_{\text {in }}-\tilde{t}\left(z, t_{0}\right)}}{\pi} \int_{t_{\text {in }}}^{\infty} \mathrm{d} t^{\prime} \frac{\ln \left|\mathscr{O}\left(t^{\prime}\right)\right|}{\sqrt{t^{\prime}-t_{\text {in }}}\left(t^{\prime}-\tilde{t}\left(z, t_{0}\right)\right)}\right) .
$$

Some rigorous properties of the bounds can be established: they are independent of the parameter $t_{0}$ in the conformal mappings (3.1) or (3.10) and remain the same if the $\leq \operatorname{sign}$ in (2.6) or (3.7) is replaced by the equality sign. Moreover, the bounds depend in a monotonous way on the parameter $I\left(I^{\prime}\right)$, in the sense that a larger value of $I\left(I^{\prime}\right)$ gives weaker constraints. An important property of the solutions to Problems 2 and 3 is that the bounds do not depend on the arbitrary phase $\delta(t)$ for $t>t_{\text {in }}$ used as input in the Omnès function (3.8), if it is sufficiently smooth [13,25].

One can show also that by varying the weight $\rho(t)$ in (2.6) or (3.7), when the weight is at our disposal, we can approach the more stringent bounds given by the equivalent conditions formulated in the stronger $L^{\infty}$-norm [4], for instance as $\|F\|_{L^{\infty}} \equiv \sup _{t>t_{+}}|F(t)| \leq I$ instead of (2.6). The strategy for the choice of $\rho(t)$ in such cases is to make a compromise between choices that lead to strong bounds and the need to ensure a precise calculation of the parameters $I$ in (2.6) or $I^{\prime}$ in (3.7).

A hierarchy of the extremal problems can be established: a rigorous result is that the bounds obtained from Problem 2 are stronger than those obtained from Problem 1. In some cases, Problem 3 leads to results much stronger than Problem 2, because it implements explicitly through the Omnès function the Riemann sheets of the elastic branch-point [13, 25].

From (3.5) it follows that interpolation theory correlates exact values of $F(t)$ at points inside the analyticity domain. However, the values used as input in practical applications are in general known with limited accuracy, and at least a part of the error is statistical. A nontrivial question is how to merge the statistical errors with the formalism of bounds. A natural strategy is to vary the input values inside the error bars and take the weakest bounds (i.e. the union of the individual allowed domains for the output variables obtained with specific input values). Then it is reasonable to attach to the predicted allowed domains the probability of the input values to be within their quoted error intervals. Some illustrations are given in the next section. 


\section{Applications}

Applications of the techniques discussed above were reported for $B \rightarrow D^{(*)} \ell v$ decays $[9,11$, 12], $K_{\ell 3}$ form factors [14, 15, 18, 19], $B \pi$ form factors [10, 16, 17], $D \pi$ form factors [20] and the pion electromagnetic form factor $[13,21,22,23,24]$. I mention that the list of references is incomplete and I apologize for the omissions due to lack of space. More references are given in the reviews $[25,26]$ and the papers quoted above.

The applications can be grouped into several types, which I shall illustrate with a few examples (for results on $K \pi$ form factors see [27]).

\subsection{Constraints on the low energy shape parameters}

The shape parameters are defined by the Taylor series (1.5) or (1.6). They are of interest for testing the expansions of ChPT, performing fits of semileptonic decay data and the precise determination of the elements of the CKM matrix in flavour physics. Rigorous constraints on these parameters have been derived for the pion electromagnetic form factor and for the $B D^{(*)}, D \pi$ and $K \pi$ form factors.

We illustrate first this application with the $D \pi$ form factors [20]. This is a typical application of Okubo approach, where the condition (2.6) is obtained from the perturbative QCD/OPE calculation of the heavy-light polarization function. Results for several moments which generalize the correlators defined in (2.2) and (2.3) are available in the literature, leading to independent constraints on the form factors (see [20]). In Figs. 4.1 we show the allowed domain for the slope $\lambda_{0}^{\prime}$ and curvature $\lambda_{0}^{\prime \prime}$ of the $D \pi$ scalar weak form factor (with the scale $M$ in (1.6) taken as $M_{\pi}$ ). The left panel illustrates the increase of the constraining power when various pieces of input are introduced successively: the large ellipse is obtained with the solution to Problem 1 using only the integral condition (2.6) and the normalization $f_{0}(0)=0.67 \pm 0.1$, the intermediate ellipse is obtained by solving Problem 2 with a model for the phase in the elastic region, and the small ellipse is obtained by using in addition a low energy theorem of Callan-Treiman (CT) type, $f_{0}\left(M_{D}^{2}-M_{\pi}^{2}\right)=1.58 \pm 0.07$. In the right panel we show the small ellipses obtained with three integral conditions of the type (2.6). The allowed domain is the intersection of the three domains. The point corresponds to a recent pole fit.
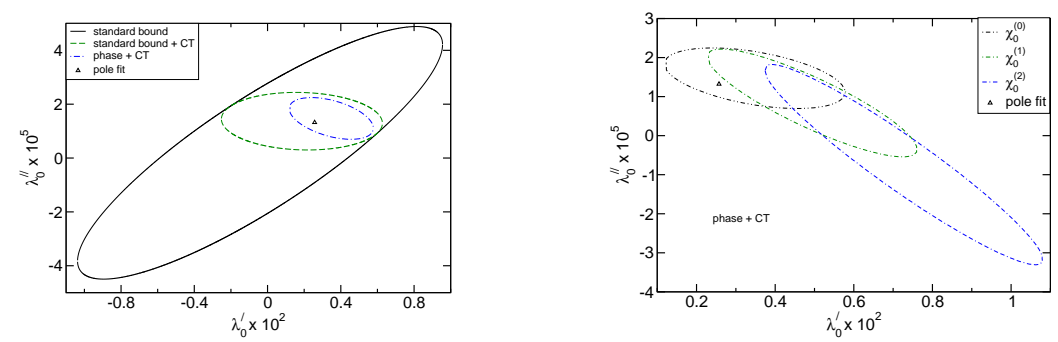

Figure 1: Allowed domain for the slope $\lambda_{0}^{\prime}$ and curvature $\lambda_{0}^{\prime \prime}$ of the scalar $D \pi$ form factor.

Very strong constraints on the low energy parameters are obtained for the pion electromagnetic form factor [13, 21, 23, 24]. In this case a very rich and precise information exists on the unitarity cut: the phase below the first important inelastic threshold $t_{\text {in }}=\left(M_{\omega}+M_{\pi}\right)^{2}$ is known from FermiWatson theorem (1.4) and the precise calculation of the phase shift $\delta_{1}^{1}$ of the $P$-wave of $\pi \pi$ elastic 
scattering from ChPT and dispersive (Roy) equations, while the modulus has been measured from high statistics data on $e^{+} e^{-} \rightarrow \pi^{+} \pi^{-}$annihilation by several experimental groups, in particular up to $3 \mathrm{GeV}$ by BaBar collaboration. Moreover, a few precise measurements at spacelike momenta $t<0$ are now available. It turns out that the most suitable way to exploit this information is based on Problem 3 of Section 3, with a suitable choice of the weight $\rho(t)$ in (3.7), which allows a precise calculation of the integral $I^{\prime}$. As discussed in detail in [23], this strongly constrains the behaviour of the form factor in the elastic region of the unitarity cut, below the $\omega \pi$ threshold, leading to predictions more precise than the data at low energies and providing nontrivial consistency tests of various data sets. Strong constraints are obtained also for the shape parameters defined in (1.5): a precise prediction for the charge radius is obtained without any specific parametrization [24]:

$$
\left\langle r_{\pi}^{2}\right\rangle=(0.43 \pm 0.01) \mathrm{fm}^{2},
$$

while the next coefficients in (1.5) are restricted to the ranges $3.8 \mathrm{GeV}^{-4} \lesssim \mathrm{c} \lesssim 4.1 \mathrm{GeV}^{-4}$ and $10.3 \mathrm{GeV}^{-6} \lesssim \mathrm{d} \lesssim 10.6 \mathrm{GeV}^{-6}$, with a strong correlation among them [21, 24].

\subsection{Zeros}

The knowledge of the possible zeros of the form factors is important for testing symmetry properties and in specific dispersion relations like the phase (1.8) and modulus (1.9) representations. The formalism discussed here can be used to define domains on the real axis and in the complex $t$-plane where zeros are excluded. To see this, insert the value $F\left(t_{c}\right)=0$ in the determinant (3.5), adding this assumption to the known input values. Obviously, if the inequality (3.5) is violated, the assumption that a zero is present is wrong. One obtains in this way a rigorous description of the domains where zeros are forbidden. For illustration we present in Figure 4.2 such domains obtained for the $D \pi$ scalar form factor and the pion electromagnetic form factor, with the input discussed in Section 4.1.
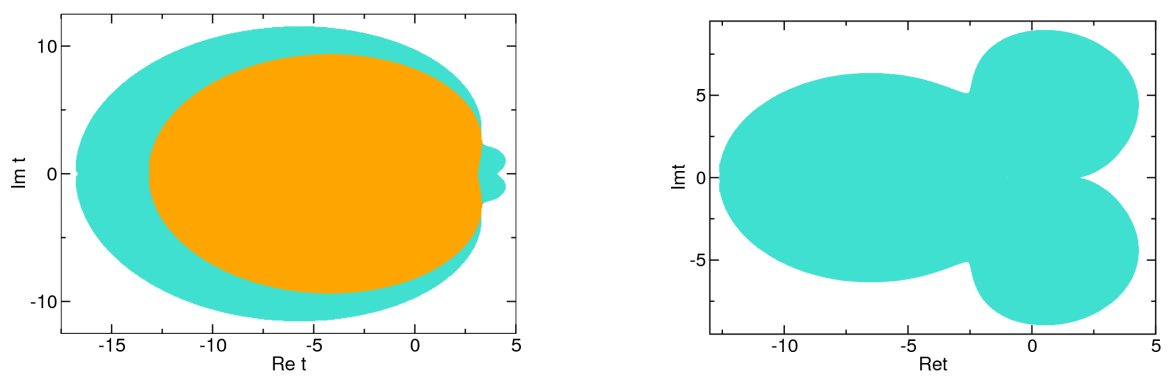

Figure 2: Left: domain in the complex $t$-plane without zeros for the scalar $D \pi$ form factor $f_{0}(t)$; right: domain without zeros for the pion electromagnetic form factor $\left(t\right.$ in $\left.\mathrm{GeV}^{2}\right)$.

\subsection{Extrapolation to spacelike energies}

The input for the pion vector form factor used in Section 4.1 was exploited in [22] for deriving model-independent upper and lower bounds on the form factor at spacelike momenta $Q^{2}=-t>0$. They are of interest for establishing the onset of the asymptotic regime of perturbative QCD, which is expected to be rather slow in this case due to the complicated interplay between the soft and the hard dynamics. 
In Fig. 4.3 we present upper and lower bounds on the product $Q^{2} F\left(-Q^{2}\right)$ derived from the solution of Problem 3, using the weight $\rho(t)=1 / \sqrt{t}$. The inner white region denotes the allowed domain delimited by the upper and lower bounds obtained with the central values of the input, while the cyan bands show the enlarged alowed domain, obtained by varying the input quantities inside their error intervals. One can see that perturbative QCD to LO is excluded for $Q^{2}<7 \mathrm{GeV}^{2}$, and perturbative QCD to NLO is excluded for $Q^{2}<6 \mathrm{GeV}^{2}$, respectively. Among the theoretical models, some are consistent with the bounds, while others are in slight disagreement at large energies (for details see [22])

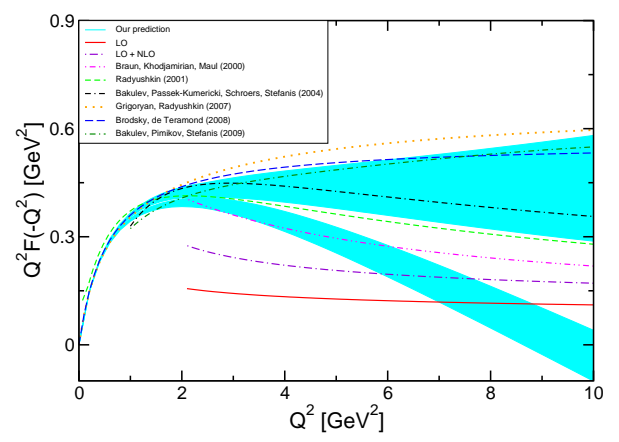

Figure 3: Bounds on $Q^{2} F\left(-Q^{2}\right)$ along the spacelike axis, compared with perturbative QCD and several nonperturbative models for the pion electromagnetic form factor.

\subsection{Analytic parametrizations with unitarity constraints}

The techniques presented above are useful also for controlling the free parameters and the truncation errors of the specific expansions of weak form factors in the physical region of semileptonic decays. The method was applied to the $B D^{(*)}$ form factors $[11,12]$ and the $B \pi$ vector form factor [17].

I briefly discuss the vector form factor $f_{+}\left(q^{2}\right)$ of $B \rightarrow \pi \ell v$ semileptonic decay, of interest for the determination of the element $\left|V_{u b}\right|$ of the CKM matrix. The parametrization [17]

$$
f_{+}\left(q^{2}\right)=\frac{1}{\left(1-q^{2} / M_{B^{*}}^{2}\right)} \sum_{k=0}^{K} b_{k}\left(t_{0}\right) z^{k}, \quad z=\tilde{z}\left(q^{2}, t_{0}\right)
$$

where $M_{B^{*}}<M_{B}+M_{\pi}$, implements correctly analyticity and QCD asymptotic scaling. The standard Okubo approach gives a quadratic condition on the free coefficients $b_{k}\left(t_{0}\right)$ :

$$
\sum_{j, k=0}^{K} B_{j k}\left(t_{0}\right) b_{j}\left(t_{0}\right) b_{k}\left(t_{0}\right) \leq 1,
$$

where $B_{j k}\left(t_{0}\right)$ are calculated in [17]. From the inequality (4.3) one can obtain an estimate of the truncation error, defined as the magnitude of the first neglected term in the expansion (4.2). Based on this, a strategy for controlling the total error was adopted in [17]: the number $K$ of terms in (4.2) was increased until the systematic error became negligible in the entire semileptonic region $0<q^{2}<\left(M_{B}-M_{\pi}\right)^{2}$ (this was achieved with $K=3$ in (4.2)). Figure 4.4 shows the result of the fit of semileptonic data and QCD sum rules and lattice calculations, for the optimal mapping $t_{0}=\left(M_{B}+M_{\pi}\right)\left(\sqrt{M_{B}}-\sqrt{M_{\pi}}\right)^{2}$ and the corresponding constraint (4.3). It leads to the prediction $\left|V_{u b}\right|=(3.54 \pm 0.30) \times 10^{-3}[17]$. 

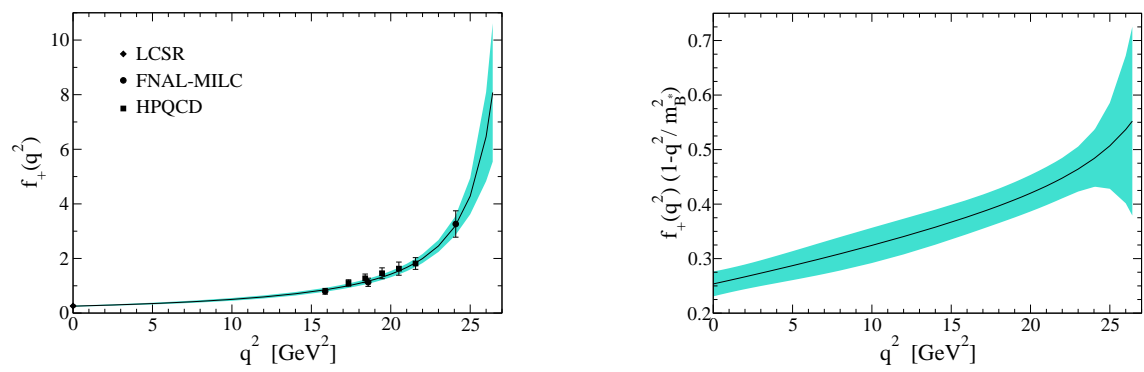

Figure 4: $B \pi$ vector form factor determined from the constrained fit, shown with points from sum-rules (LCSR) and lattice input. Right: numerator of the optimal parametrization. The band is the statistical error.

\section{Conclusions}

In this talk I presented more sophisticated analytic techniques that might be an useful alternative to the standard dispersion representations (1.7), (1.8) and (1.9), and to the specific parametrizations usually adopted for the hadronic form factors. These techniques allow a more conservative implementation of the available input on the modulus and the phase on the unitarity cut, avoiding ad-hoc assumptions often adopted in standard approaches. Experimental data, low-energy theorems of ChPT and lattice calculations at points inside the holomorphy domain provide a further valuable input in the formalism. The price to be paid for the greater model-independence is the fact that one can derive only upper and lower bounds on the quantities of interest, instead of making definite predictions. However, due to the increased accuracy of the input, the bounds are often very stringent, competing in precision with experiment and theoretical predictions. Therefore, the formalism proves to be a strong tool for precison predictions on hadronic form factors at low energies.

Acknowledgments: I thank my collaborators G. Abbas, B. Ananthanarayan, C. Bourrely, D. Das, I. Sentitemsu Imsong, L. Lellouch and S. Ramanan, whose work I used in this talk. Support from Romanian CNCS in the Program Idei-PCE, Contract No. 121/2011 is acknowledged.

\section{References}

[1] S. Ciulli, C. Pomponiu and I. Sabba-Stefanescu, Analytic extrapolation techniques and stability problems in dispersion relation theory, Phys. Rept. 17 (1975) 133.

[2] S. Okubo, Exact bounds for $K_{\ell 3}$ decay parameters, Phys. Rev. D 3 (1971) 2807.

[3] N.N. Meiman, Analytic expressions for upper limits of coupling constants in quantum field theory, Sov. Phys. JETP 17 (1963) 830.

[4] P. Duren, Theory of $H^{\mathrm{p}}$ Spaces, Academic Press, New York, 1970.

[5] M. Micu, Bounds for K K ${ }_{\ell 3}$ decay parameters, Nucl. Phys. B 44 (1972) 531.

[6] G. Auberson, G. Mahoux, F.R.A. Simão, On the bounds for the $K_{\ell 3}$ form-factors, Nucl. Phys. $B \mathbf{9 8}$ (1975) 204.

[7] V. Singh and A.K. Raina, Bounds on form-factors and propagators, Fortsch. Phys. 27 (1979) 561.

[8] C. Bourrely, B. Machet and E. de Rafael, Semileptonic decays of pseudoscalar particles $\left(M \rightarrow M^{\prime}+\ell+v_{\ell}\right)$ and short distance behavior of quantum chromodynamics, Nucl. Phys. B 189 (1981) 157. 
[9] E.de Rafael and J. Taron, Constraints on heavy meson form-factors, Phys. Lett. B 282 (1992) 215.

[10] L. Lellouch, Lattice-constrained unitarity bounds for $\bar{B}^{0} \rightarrow \pi^{+} \ell \bar{v}_{\ell}$ decays, Nucl. Phys. B 479 (1996) 353 [hep-ph/9509358].

[11] C.G. Boyd, B. Grinstein and R.F. Lebed, Model independent determinations of $B \rightarrow D^{(*)} \ell \bar{v}$ form factors, Nucl. Phys. B 461 (1996) 493 [hep-ph/9508211].

[12] I. Caprini, L. Lellouch and M. Neubert, Dispersive bounds on the shape of $\bar{B} \rightarrow D^{(*)} \ell \bar{v}$ form factors, Nucl. Phys. B 530 (1998) 153 [hep-ph / 9712417$].$

[13] I. Caprini, Dispersive and chiral symmetry constraints on the light meson form-factors, Eur. Phys. J. C 13 (2000) 471 [hep-ph / 9907227$].$

[14] C. Bourrely and I. Caprini, Bounds on the slope and the curvature of the scalar $K \pi$ form factor at zero momentum transfer, Nucl. Phys. B 722 (2005) 149 [hep-ph/ 0504016 ].

[15] R.J. Hill, Constraints on the form factors for $K \rightarrow \pi \ell v$ and implications for $\left|V_{u s}\right|$, Phys. Rev. D 74 (2006) 096006 [hep-ph / 0607108$].$

[16] T. Becher and R.J. Hill, Comment on form factor shape and extraction of $\left|V_{u b}\right|$ from $B \rightarrow \pi \ell v$, Phys. Lett. B 633 (2006) 61 [hep-ph/ 0509090$].$

[17] C. Bourrely, I. Caprini and L. Lellouch, Model-independent description of $B \rightarrow \pi \ell v$ decays and $a$ determination of $\left|V_{u b}\right|$, Phys. Rev. D 79 (2009) 013008 [arXiv:0807.2722 [hep-ph] ].

[18] G. Abbas, B. Ananthanarayan, I. Caprini, I.Sentitemsu Imsong and S. Ramanan, Stringent constraints on the scalar $K \pi$ form factor from analyticity, unitarity and low-energy theorems, Eur. Phys. J. A 44 (2010) 175 [arXiv:0912.2831 [hep-ph]].

[19] G. Abbas, B. Ananthanarayan, I. Caprini and I.Sentitemsu Imsong, Improving the phenomenology of $K_{\ell 3}$ form factors with analyticity and unitarity, Phys.Rev. D 82 (2010) 094018 [arXiv: 1008.0925 [hep-ph]].

[20] B. Ananthanarayan, I. Caprini and I. Sentitemsu Imsong, Implications of unitarity and analyticity for the D $\pi$ form factors, Eur. Phys. J. A 47 (2011) 147 [arXiv:1108.0284 [hep-ph]].

[21] B. Ananthanarayan, I. Caprini and I.S. Imsong, Implications of the recent high statistics determination of the pion electromagnetic form factor in the timelike region, Phys. Rev. D 83 (2011) 096002 [arXiv: 1102.3299 [hep-ph]].

[22] B. Ananthanarayan, I. Caprini and I.S. Imsong, Spacelike pion form factor from analytic continuation and the onset of perturbative QCD, Phys. Rev. D 85 (2012) 096006 [arXiv: 1203.5398 [hep-ph]].

[23] B. Ananthanarayan, I. Caprini, D. Das and I.S. Imsong, Model independent bounds on the modulus of the pion form factor on the unitarity cut below the $\omega \pi$ threshold, Eur. Phys. J. C 72 (2012) 2192 [arXiv:1209.0379 [hep-ph]].

[24] B. Ananthanarayan, I. Caprini, D. Das and I.S. Imsong, Parametrization-free determination of the pion charge radius, in preparation.

[25] G. Abbas, B. Ananthanarayan, I. Caprini, I.S. Imsong and S. Ramanan, Theory of unitarity bounds and low energy form factors, Eur.Phys. J. A 45 (2010) 389 [arXiv: 1004.4257 [hep-ph] ].

[26] B. Ananthanarayan and I. Caprini, Constraining form factors with the method of unitarity bounds, J. Phys. Conf. Ser. 374 (2012) 012011 [arXiv:1202.5391 [hep-ph] ].

[27] G. Abbas, B. Ananthanarayan, I. Caprini, I. Sentitemsu Imsong, Unitarity and analyticity constraints on the $K \pi$ form factors, these Proceedings. 